\title{
Valoración económica del almacenamiento de agua y carbono en la comunidad campesina Villa de Junín
}

\section{Economic estimation of water and carbon storage in the Villa Junín rural community}

\author{
Miguel Ángel Vila Balbin \\ Universidad Continental \\ MIKI0073@hotmail.com
}

\author{
Liz Amelia Chupan Minaya² \\ Universidad Continental \\ liz d7@hotmail.com
}

\section{RESUMEN}

El objetivo ha sido estimar el valor económico que generan los bofedales en el almacenamiento de agua y carbono en la comunidad campesina Villa de Junín en el departamento de Junín, Perú. El proceso de recolección de datos a estado enfocado en datos meteorológicos (temperatura y precipitación), datos de la actividad económica predominante (ganadería), recolección de muestras de suelo para hallar el contenido de carbono y capacidad volumétrica; posteriormente se hizo la comparación del beneficio percibido por la actividad ganadera y el beneficio que se podría obtener por los servicios que brinda un bofedal (almacenamiento de agua y carbono). Entre los resultados respecto a la precipitación anual se ha determinado 22367664 m3/año, del cual un 42,56 \% (9 294 933,67 m3/ año) regresa a la atmósfera a través del proceso de la evapotranspiración, quedando una oferta hídrica disponible de 13072 730,33 m3/año, que representa un $57,44 \%$ de la oferta hídrica total; la productividad hídrica es de 0,01 S/./m3. El costo de oportunidad que tiene la ganadería es de 200,38 S/./ha /año; el valor económico de agua y carbono es de S/. 48974 181,79 y S/. 44305010,31 respectivamente. Se concluye que el almacenamiento de agua y carbono brindan mayores ingresos económicos a la población que la actividad ganadera.

Palabras clave: Carbono, capacidad volumétrica, precipitación, evapotranspiración, productividad hídrica, costo de oportunidad.

\section{ABSTRACT}

The objective is to estimate the economic value that the wetlands generate in the water and carbon storage in the "Villa de Junín" rural community in the Junín department, Perú. The data collection process was based on the metereological data (weather and rainfall), some data of the main economic activity (cattle industry), soil samples to find out the carbon content and volumetric capacity; later we have made the gotten benefit comparison from the cattle industry with the benefit that might be gotten through the wetland services (water and carbon storage). As a result, there is an annual rainfall of 22367 $664 \mathrm{~m} 3 /$ year, from which a 42,56 \% (9 294933,67 $\mathrm{m} 3$ /year) goes back to the atmosphere through the evapotranspiration process, remaining an available hydrological offer of 13072730,33 m3/year, which represents a $57,44 \%$ of the whole hydrological offer; the hydrological production of $0,01 \mathrm{~S} / . / \mathrm{m} 3$. The opportunity cost of the cattle industry is 200,38 $\mathrm{S} /$. /ha /year; the water and carbon economic value is of S/. 48974 181,79 and S/. 44305 010,31 respectively. It is concluded that the water and carbon storage provides better economical incomes to the population than the cattle industry.

Keywords: Carbon, volumetric capacity, rainfall, evapotranspiration, hydrological production, opportunity cost.

Historial del artículo:

Recibido: 27 de septiembre de 2014. Aprobado: 5 de febrero de 2015. Disponible en línea: 30 de diciembre de 2015

1 Ingeniero en Ingeniería Forestal y Ambiental.

2 Ingeniera en Ingeniería Forestal y Ambiental. 


\section{INTRODUCCIÓN}

Los bofedales son formaciones vegetales que se desarrollan en ambientes edáficos principalmente orgánicos conocidos como turberas naturales altoandinas (1). Este ecosistema tiene una importancia ecológica, económica y sociocultural (2); debido a esta importancia los bofedales proveen bienes y servicios como: almacenamiento y regulación del régimen hídrico, almacenamiento de carbono en la flora y suelo, refugio de flora y fauna endémica (3).

Pero estos bienes y servicios que ofrecen los bofedales $u$ otro recurso natural no son valorados por el hombre debido al desconocimiento o poco interés que existe en asignarles valores cuantitativos expresados en valores monetarios de los bienes y servicios que ofrece un recurso natural, si se tuviera una valoración económica de los recursos naturales que poseemos nos permitiría medir y comparar los distintos beneficios que ofrecen y así servir como un instrumento eficaz de facilitación y mejoramiento del uso racional y manejo o gestión de los recursos naturales (4).

El lago Chinchaycocha (humedal de importancia internacional declarado por la convención internacional RAMSAR) está formado por tres tipos de flora: los bofedales, los pajonales y los totorales. De ellos los bofedales son utilizados por la población de varias comunidades campesinas que están alrededor por ejemplo la Comunidad Campesina Villa de Junín donde se encuentra la mayor extensión de bofedales se realiza actividades como: ganadería (sobrepastoreo), quema de pastos, extracción de pastos (recurso energético) que están alterando este tipo de ecosistema (debido a la compactación del suelo y disminución de la cobertura vegetal) y con ello los bienes y servicios que brinda (5).

En este estudio se ha planteado como objetivo la estimación del valor económico que generan los bofedales en la comunidad campesina Villa de Junín en el almacenamiento de agua y carbono, y ha permitido determinar que constituyen una fuente de ingreso económico importante para la población asentada en la mencionada comunidad mejor inclusive que la dedicación a otras actividades económicas como es el caso de la ganadería en la cual participa un alto porcentaje de los comuneros.

\section{MATERIAL Y MÉTODOS}

\section{Área de estudio}

La comunidad campesina Villa de Junín se ubica en el distrito de Junín a una altitud de 4105 m s.n.m., posee una extensión de 2673 ha de bofedales, posee un clima húmedo-frígido. La temperatura máxima promedio es de $7^{\circ} \mathrm{C}$ y la temperatura mínima es de $5{ }^{\circ} \mathrm{C}$, la precipitación es irregular durante todo el año presentándose con mayor frecuencia en los meses de octubre a marzo y menos frecuente en los meses de mayo a agosto. Posee una zona de vida Paramo muy Húmedo - Subalpino Tropical. La pendiente del área de estudio es plana (6).

El área que ocupan los bofedales dentro de la comunidad campesina Villa de Junín es de 2673 ha donde se delimitaron 5 parcelas de 1 ha, de cada parcela se extrajo 30 muestras de suelo para determinar el carbono almacenado, éstas muestras fueron enviadas al laboratorio del Instituto Nacional

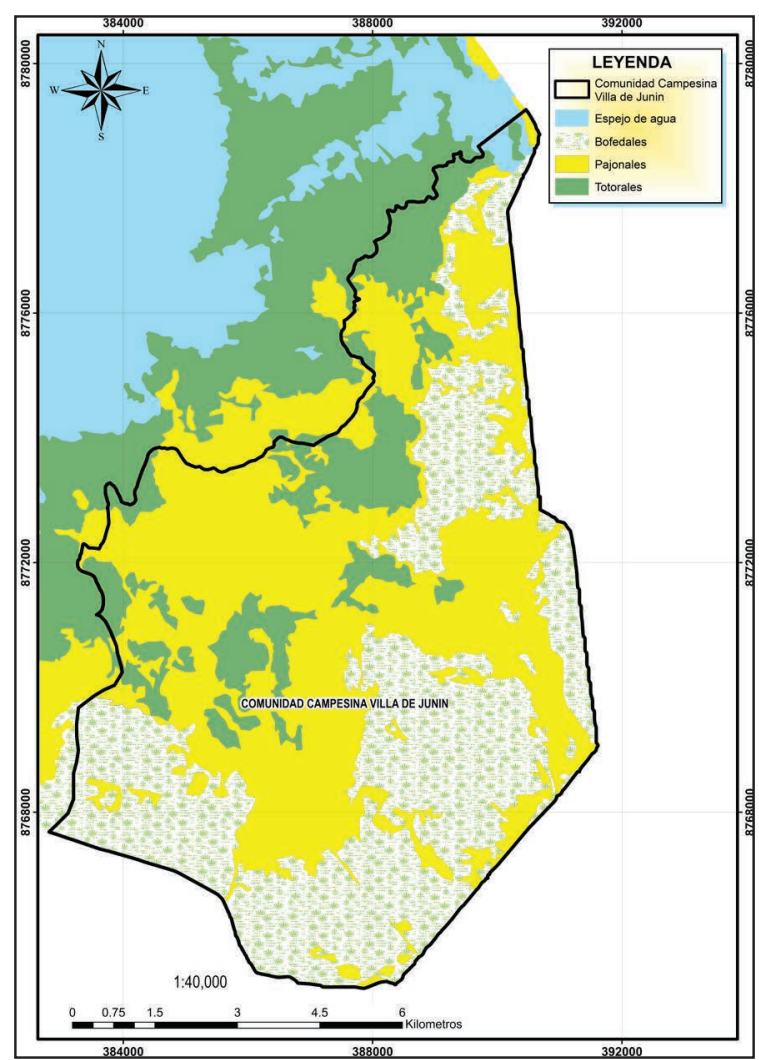

Figura $\mathrm{N}^{\circ} 1$ : Mapa de ubicación de la comunidad campesina Villa de Junín.

de Investigación Agraria (INIA) para la determinación del carbono. De estas muestras también se determinó la capacidad volumétrica que tienen los suelos de los bofedales.

Dentro del área de estudio se encuentran a 150 jefes de familia que se dedican a la ganadería actividad principal que se realiza en el área de los bofedales, se realizó una encuesta a 108 jefes de familia para determinar el costo de oportunidad que tiene la ganadería (nivel de confianza de $95 \%$ y error del $5 \%$ ). 


\section{Determinación del valor económico del almacenamiento de agua}

Se utilizaron datos de temperatura y precipitación registrados durante los últimos 50 años en la estación meteorológica de Upamayo (7), también datos que se recolectaron de campo, como el nivel freático y costo de oportunidad.

a. Valor económico del almacenamiento de agua: $\mathrm{V}_{\text {(almacenamiento agua) }}=\partial * \mathrm{~A} * \rho *(0,01) * \mathrm{C}_{\text {(unit represa) }}$

Donde:

$\mathrm{V}=$ Es el valor en dólares (\$) del servicio ambiental hídrico de almacenamiento de agua

$\partial=$ Es el porcentaje de almacenamiento de agua en el suelo de los bofedales

A = Es la superficie de bofedales medida en ha dentro la zona de estudio

$\rho=$ Es el nivel freático del suelo medido en $\mathrm{cm}$ para la zona de bofedales

$\mathrm{C}=$ Es el costo unitario en $(\$ / \mathrm{m} 3)$ para una represa.

b. Estimación del valor económico de la Productividad Hídrica

$\mathrm{VP}=\sum_{i=1}^{\mathrm{n}} \frac{\alpha \mathrm{i} \beta \mathrm{i} \text { Abi }}{\text { Odi }}$

Donde:

$\mathrm{VP}=$ Valor de productividad hídrica del bofedal secundario $(\$ / \mathrm{m} 3)$

$\beta \mathrm{i}=$ Costo de oportunidad de la ganadería (\$/ha/ año)

Abi = Área bajo cobertura de bofedal secundario en la zona de Estudio (ha)

Odi $=$ Volumen de agua disponible captada por los bofedales (m3/año)

$\alpha i=$ Índice de Protección Hidrológica

c. Estimación de la Oferta Total Hídrica

$\mathrm{OT}=\sum_{\mathrm{i}=1}^{\mathrm{n}} \mathrm{Pi} \times \mathrm{Ai}$

Donde:

OT = Oferta total hídrica en el área de importancia

\author{
(m3/año) \\ $\mathrm{Pi}=$ Precipitación en el bofedal i (mm/año) \\ $\mathrm{n}=$ Número de bofedales \\ $\mathrm{Ai}=$ Área del bofedal i (m2)
}

\section{Determinación del valor económico del almacenamiento de carbono en el suelo}

Para la determinación del carbono, se necesitó el suelo ser medido hasta una profundidad de $30 \mathrm{~cm}$, debido a que el cambio de uso de tierra tiene mayor efecto en los estratos superiores. La muestra llevada a laboratorio corresponde a un $\mathrm{kg}$ del suelo homogenizado a partir de la mezcla de todas las unidades muéstrales tomadas para cada estrato.

a. Valor económico de almacenamiento de carbono en el suelo

Vcarbono $=$ Contenido de $\mathrm{C}$ en bofedales $\left(\frac{\mathrm{t} \mathrm{CO} 2}{\text { ha }}\right) *$ Costo social del $\mathrm{C}\left(\frac{\$}{{ }_{\mathrm{t}} \mathrm{CO} 2}\right)$

b. Carbono almacenado en el suelo

$\mathrm{C}=$ Ps $\times \mathrm{M}$

Donde:

$$
\begin{aligned}
& C=\text { Carbono total capturado }+\mathrm{C} / \mathrm{ha} \\
& \text { Ps }=\text { Peso del suelo } \\
& M=\text { Carbono (expresado sin porcentaje) }
\end{aligned}
$$

c. Dióxido de carbono almacenado en el suelo

$\mathrm{CO} 2=\mathrm{C} \times \mathrm{Kr}$

Donde:

$\mathrm{CO} 2=\mathrm{T}$ de dióxido de carbono $(\mathrm{t} / \mathrm{CO} 2)$

$\mathrm{C}=$ Carbono

$\mathrm{Kr}=44 / 12$ (Este factor proviene de la relación de estequiometria de pesos moleculares con la finalidad de conocer cuánto $\mathrm{CO} 2$ puede originarse porla descomposición del carbono orgánico).

\section{Determinación del costo de oportunidad}

Se realizó a través de encuestas socioeconómicas, para ello la actividad predominante que compite con el suelo del suelo de los bofedales es la ganadería, con el costo de oportunidad se determina la rentabilidad que existe al renunciar a una actividad en este caso a la ganadería para conservar y mantener el uso del suelo como bofedal. 
$\mathrm{CO}=\frac{\sum_{(\mathrm{i}=1)}^{\mathrm{n}}(\mathrm{Ytx}-\mathrm{Ctx})^{\mathrm{n}}+\sum_{(\mathrm{i}=1)}(\mathrm{Yax}-\mathrm{Cpx})}{\mathrm{A}}$

Donde:

$Y+x=$ Ingreso total por la venta del producto $\mathrm{i}$

$\mathrm{Ctx}=$ Costos efectivos de producción de i para la venta

Yax $=$ Ahorro por autoconsumo del producto $\mathrm{i}$

Cpx = Costo de producción de i para autoconsumo

$A=$ Superficie de pastoreo de la ganadería

\section{RESULTADOS}

\section{Valor económico del almacenamiento de agua}

\section{Costo de oportunidad}

El costo de oportunidad promedio que tiene la ganadería en la comunidad campesina Villa de Junín es de S/. 220,38 al año por ha. Este valor proviene de los jefes de familia que pastorean sus ganados en el área de los bofedales, el costo de oportunidad del ganado vacuno fue mayor que el costo de oportunidad para el ganado ovino, existiendo una distribución amplia de variabilidad (desviación estándar igual a 508,32 y 59,11 para ganado vacuno y ovino respectivamente) esto se debe a las variaciones en la posesión de animales (existe mayor posesión de ganado vacuno que de ganado ovino) que tiene cada jefe de familia. Según las encuestas realizadas los jefes de familia reciben mayores beneficios económicos del ganado vacuno (figura 2 ).

\section{Oferta hídrica disponible}

La precipitación anual promedio en la estación de Upamayo es de $836,8 \mathrm{~mm}$ al año para el periodo histórico 1965 - 2005, entonces en el área de estudio se precipita en promedio anual de 22367 $664 \mathrm{~m} 3$, del cual un 42,56 \% (9 $294933,67 \mathrm{~m} 3 /$ año) regresa a la atmosfera a través del proceso de la evapotranspiración, quedando una oferta hídrica disponible de 13072 730,33 m3/año que representa un $57,44 \%$ de la oferta hídrica total.

\section{Valor económico de la productividad hídrica}

Conociendo el costo de oportunidad, el área de los bofedales y la oferta hídrica disponible se calculó el valor económico de la productividad hídrica que es igual a S/. 0,01 por metro cubico, este valor es muy bajo debido a que el área está siendo alterado por la actividad ganadera que compacta y disminuye la cobertura vegetal y por consiguiente disminuye la productividad hídrica.

\section{Valor económico del almacenamiento de agua en suelos de los bofedales}

Los suelos de los bofedales en el área de estudio tienen una capacidad de almacenar agua de $65,86 \%$, la napa freática se encuentra en promedio a $0,35 \mathrm{~m}$ de la superficie, el área total de los bofedales es de 2673 ha y el dato de costo unitario para la construcción de una represa se tomó de la construcción de la represa de Yanaqocha cuyo valor es de S/. 7,95 por m3. Teniendo estos datos se tiene un valor económico de S/. 18325,55 por ha, lo cual representa un valor total para toda el área de estudio de S/. 48974 181,79.

Valor económico del almacenamiento de carbono en los suelos de los bofedales de la comunidad campesina Villa de Junín

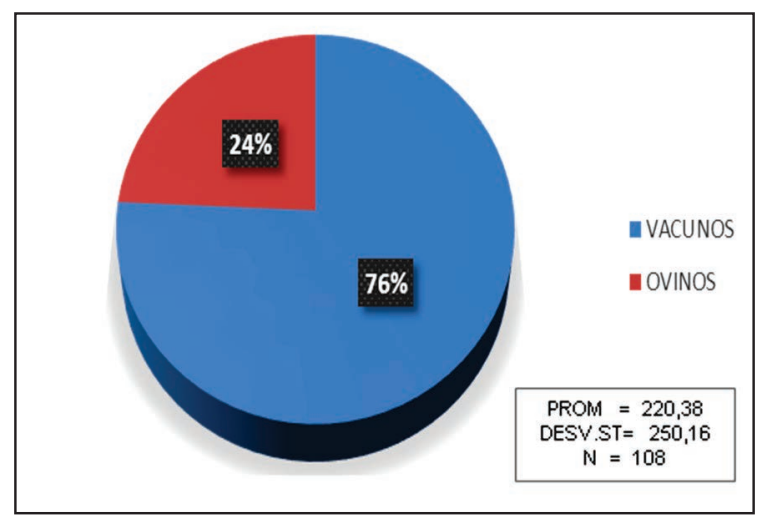

Figura $\mathrm{N}^{\circ}$ 2: Costo de oportunidad de la ganadería.

El valor de la densidad para las muestras recogidas en campo es 0,39 $\mathrm{gr} / \mathrm{cm} 3$. La profundidad a la cual se tomó las muestran fueron diferentes siendo los primeros $0,35 \mathrm{~m}$ de profundidad donde el almacenamiento es más alto. El contenido de carbono es de $25,22 \%$ en promedio para las muestras recolectadas, el costo de carbono se estimó en función al costo social que cuesta en el mercado internacional que es de $\$ 8$ por cada † de dióxido de carbono no emitido o reducido. Con estos datos el servicio ambiental de almacenamiento de carbono para los suelos de los bofedales en la comunidad campesina Villa de Junín es de S/. 16 575,01 por ha, lo cual representa un total de S/. 44 305 010,31 para toda el área de estudio.

Integración del valor económico de los servicios ambientales de almacenamiento de agua $y$ almacenamiento de carbono

En la figura 3 se observa los valores económicos del servicio ambiental en el almacenamiento de 
agua y almacenamiento de carbono siendo mayor económicamente el valor del almacenamiento de agua. Para el servicio ambiental de almacenamiento de agua la cantidad disponible de recurso hídrico es un valor de uso directo ya que es utilizada en diversas actividades como riego, consumo directo, abrevaderos, etc. (4). En cambio los servicios ambientales de almacenamiento de agua y de carbono son considerados como valores de uso indirecto ya que no son directamente consumidos sino que derivan de la protección que se le da al bofedal (4).

Por lo tanto el almacenamiento de agua en los suelos de los bofedales ayuda a la regulación del régimen hídrico (dotando de agua en los meses secos y evitando excesivos caudales en periodos intensos de lluvia); el servicio ambiental de almacenamiento de carbono evita la liberación de CO2 al ambiente el cual es considerado como servicio de regulación en el medio ambiente frente al calentamiento global (3). El servicio ambiental de almacenamiento de agua

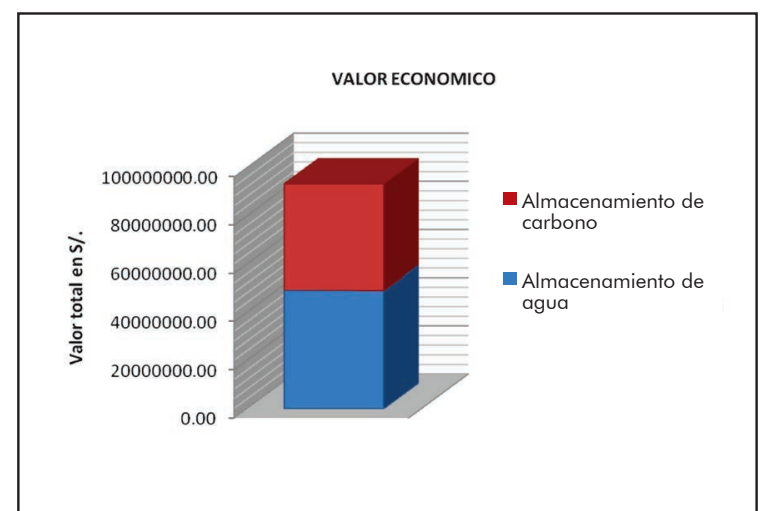

Figura $\mathrm{N}^{\circ}$ 3: Valor económico del almacenamiento de agua y carbón en los bofedales.

y el servicio ambiental de carbono tienen una valor económico total para todo el área de estudio de $\mathrm{S} /$. $93279192,10$.

\section{DISCUSIÓN}

Los bofedales de la comunidad Villa de Junín son capaces de almacenar grandes cantidades de agua que expresadas en unidades monetarias equivale a 18 325,55 S/./ha, este valor es alto en comparación al valor económico del almacenamiento de agua que ofrece el bofedal de Oña-Nabón-Saraguro-Yacuambi (bofedal no intervenido) cuyo valor es de 3 196,8 \$/ ha $(9046,94 \mathrm{~S} / . /$ ha) (8), esto se debe a la ocurrencia de niveles altos de precipitación en la comunidad Villa de Junín llegando en promedio a 836,8 mm al año en comparación a Oña-Nabón-Saraguro-Yacuambi que a pesar de ser un bofedal no intervenido registra una precipitación anual de 505,1 mm, lo cual conlleva a que la oferta hídrica sea menor y por consecuente el valor económico del almacenamiento de agua. Aun mayor es la diferencia que existe entre la valoración económica de almacenamiento de agua en el área de estudio al compararlo con la valoración económica de almacenamiento de agua en el bofedal de Tungurahua que a pesar de presentar una precipitación promedio anual de $800 \mathrm{~mm}$ anual su valor económico se reduce a solo 2 258,4 \$/ha (6 391,27 S/./ha), este bofedal está altamente intervenido por la actividad ganadera lo que ha provocado que el almacenamiento sea menor debido a la compactación y erosión que se produce aun cuando presenta una precipitación promedio anual alta. Esto demuestra que la capacidad de almacenamiento de agua que ofrecen los bofedales de la Comunidad Campesina Villa de Junín a pesar de estar siendo erosionados y compactados por la actividad ganadera no ha perdido la capacidad de ofrecer este servicio ambiental.

En relación al valor económico de la productividad hídrica, se estimó para los bofedales de Villa de Junín $0,01 \mathrm{~S} / . / \mathrm{m} 3$, valor que es bajo en comparación a los bofedales de Oña-Nabón-Saraguro-Yacuambi y Tungurahua (8) que tienen valores de productividad hídrica de 0,03 \$/m3 (equivalente a 0,09 S/./m3) y 0,02 $\$ / \mathrm{m} 3$ (equivalente a $0,06 \mathrm{~S} / . / \mathrm{m} 3$ ) respectivamente, esto se debe a que el valor de productividad hídrica esta en relación al costo de oportunidad, siendo este costo menor para la comunidad campesina Villa de Junín, lo cual reduce el valor de la productividad hídrica. Sin embargo al compararlo con el valor de productividad hídrica del bofedal de Jimbura (9) es un valor similar debido a que el costo oportunidad para esta zona es relativamente igual al costo de oportunidad para el área de estudio.

El costo estimado que se obtendría por el almacenamiento de carbono en la comunidad campesina Villa de Junín, considerando el precio de $\$ 8$ por $t$ de carbono en el mercado internacional es de $16575,01 \mathrm{~S} / . / \mathrm{ha}$, valor por debajo de los obtenidos en los bofedales de Oña-Nabón-SaraguroYacuambi y Tungurahua (8) quien estimo valores de 13340,62 \$/ha (37 753,96 S/./ha) y 7787,26 \$ ha $(22037,95 \mathrm{~S} / . /$ ha) respectivamente. Esto sucede por la disminución del precio del CO2, ya que en el año 2010 la † de CO2/ha llegaba a costar 15 pero para los últimos años este valor ha decrecido debido a la crisis económica mundial. Mayor diferencia se encuentra al compararlo con los valores obtenidos en la valoración económica de almacenamiento de carbono en los suelos de los bofedales de Mechahuasca y Paylacocha (10) donde refiere que estos tienen un valor de 1562,18 \$/ha (4 420,97 S/./ ha) y $1578,67 \$ /$ ha $(4467,64 \mathrm{~S} / . / \mathrm{ha})$, justificando estos valores por el cambio de uso que se produjeron en estas áreas, lo cual ha disminuido este servicio ambiental. Cabe mencionar que el almacenamiento 
de carbono en los suelos de los bofedales de Villa de Junín $(732,11$ +CO2/ha) es menor a almacenamiento de carbono en los suelos de los bofedales de OñaNabón-Saraguro-Yacuambi $(889,37$ + CO2/ha), esto se debe a la conservación y a la reducida intervención de las actividades humanas como la ganadería y la agricultura dentro de este último. Sin embrago el valor obtenido en el área estudiada es mayor al compararlo con el bofedal de Tungurahua donde los suelos de los bofedales llegan a almacenar 519,15 + CO2/ha esta diferencia se debe a que Tungurahua es un bofedal que presenta mayor intervención humana que los bofedales de Villa de Junín.

El costo de oportunidad estimado para el área de estudio, referente a la actividad ganadera como principal actividad económica dentro del área es de 220,38 S/./ha/año, valor inferior a los presentados para los bofedales de Oña-Nabón-Saraguro-Yacuambi y Tungurahua (8) quien estima un valor de 126,15 \$/ ha/año (357 S/./ha/año), al mismo tiempo el valor obtenido para el costo oportunidad en el área de Villa de Junín es mayor al compararlo al valor estimado para el bofedal de Jimbura (9) que es de 58,03 \$/ha/ año (164,22 S/./ha/año). Estas variaciones se deben principalmente a la tenencia de ganado, relacionado directamente con el número de familias dedicadas a la ganadería y a los beneficios económicos obtenidos por la venta de ganados y los subproductos derivados de estos.

Las principales conclusiones son:

El valor económico estimado para el servicio de almacenamiento de agua que ofrecen los bofedales de la comunidad campesina Villa de Junín es de S/.48 974181,79 lo cual expresados en valor por hectárea es de $18325,55 \mathrm{~S} / . /$ ha.

Los suelos de los bofedales de la comunidad campesina de Villa de Junín almacenan 732,11 + CO2/ha que equivale a $16575,01 \mathrm{~S} / . / \mathrm{ha}$, obteniendo un total de S/. 44305 010,31 para toda el área estudiada.

La actividad ganadera es una fuente de ingresos para los pobladores de la comunidad campesina de Villa de Junín sin embrago al analizar el costo de oportunidad que determina la rentabilidad de esta actividad se percibe que los beneficios que obtienen los pobladores dedicados a la ganadería es en promedio el valor de 220,38 S/./ha/año, que al contrastarlo con los beneficios económicos que se obtendrían por la conservación del bofedal resulta muy inferior, sabiendo que por el almacenamiento de agua y carbono se obtiene 34 900,56 S/./ha/año resultando muy rentable la conservación de los bofedales y con ello los servicios ecosistémicos de almacenamiento de agua y carbono que ofrecen.

\section{REFERENCIAS BIBLIOGRÁFICAS}

1. Carafa T. Evaluación ecológica de bofedales de la cuenca circundante al nevado Illimani. La Paz: Agua Sustentable; 2009. Informe final.

2. Alzérreca $H$, Prieto $G$, Laura J, Luna $D$, Laguna $S$. Características y distribución de los bofedales en el ámbito Boliviano. La Paz. Informe de Consultoría. Asociación Integral de Ganaderos en Camélidos de los Andes Altos (AIGACAA), Autoridad Binacional del Lago Titicaca (ALT), Gerencia del Proyecto de Biodiversidad; 2001.

3. Gil J. Bofedal: Humedal altoandino de importancia para el desarrollo de la región Cusco [Internet]. Cusco; 2011 [Citado: 10/04/13]. Disponible en: http:// www.cebem.org/cmsfiles/artículos/ Humedales_conservacion.pdff

4. Barbier E, Acreman M y Knowler D. Valoración Económica de los Humedales, guía para decisores y planificadores. Suiza: Oficina de la Convención de Ramsar; 1997.

5. Caro C. Extracción de pastos por actividad de "champeo" en la Reserva Nacional de Junín durante el año 2004 - 2005. Una perspectiva desde la teoría de la sucesión: estudio de caso en la comunidad campesina de Villa Junín (Tesis para optar el grado de doctor).Lima: Universidad Nacional Agraria La Molina; 2010.

6. Intendencia de Áreas Naturales Protegidas. Plan Maestro de la Reserva Nacional de Junín 20082012 - Documento de trabajo [Internet]. Lima: Instituto Nacional de Recursos Naturales; 2009 [citado 08/09/11]. Disponible en: http://www. sernanp.gob.pe/sernanp/archivos/biblioteca/ publicaciones/RN_Junin/PlanMaestro2008-2012RNJunin.pdf

7. Barrantes G y Vega M. Evaluación del servicio ambiental hídrico en la cuenca del rio Savegre con fines de ordenamiento territorial. Costa Rica: Instituto de Políticas para la Sostenibilidad; 2001.

8. Castro, M. Una valoración económica del almacenamiento de agua y carbono en los bofedales de los páramos ecuatorianos - la experiencia en Oña-Nabón-Saraguro-Yacuambi y el Frente Suroccidental de Tungurahua. EcoCiencia / Wetlands International / UTPL / MAE. Quito: Integraf. 2011

9. Castro M. Valoración económica del agua de los bofedales de las lagunas Negras de Jimbura, Nudo de Sabanilla. Quito: EcoCiencia/ Ministerio del Ambiente del Ecuador. Quito: Integraf. 2009.

10. Villares E. Valoración Económica de agua y carbono almacenado en los bofedales de Mechahuasca y Paylacocha en la reserva Chimborazo (Tesis para optar el grado de Ingeniero Forestal). Ecuador: Escuela Superior Politécnica de Chimborazo; 2012. 